\title{
La fusión de cooperativas: ¿el mejor mecanismo de integración?
}

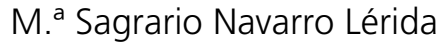 \\ Profesora Contratada Doctora de Mercantil - UCLM
}

Sumario: 1. La integración cooperativa como principio y los mecanismos de integración. 2. La fusión de las cooperativas agrarias y las entidades asociativas prioritarias: ¿fomento ex lege u obligación?. 3. Principales «problemas» de la fusión de cooperativas 3.1. La relación de canje y a patrimonialización de la posición del socio. 3.2. El derecho de separación: su fundamento y la analogía con su utilización en las sociedades de capital. 3.3. Las cuestiones del derecho de la competencia. 4. Conclusiones. Bibliografía

Resumen: La integración cooperativa late en la naturaleza de esta forma societaria y enlaza con los principios cooperativos. Sin embargo, los mecanismos a través de los cuales puede realizarse con variados. En este trabajo se analizan las fortalezas y debilidades de una de esas fórmulas: la fusión. Tomando como punto de partida la actual situación del sector agroalimentario y los incentivos legales que estos procesos de concentración tienen, a través de la creación de las llamadas Entidades Asociativas Prioritarias, y las normas contenidas en la Ley de medidas para la mejora de la cadena alimentaria.

Palabras clave: Cooperativas, fusión, mecanismos de integración

Abstract: The cooperative integration exist in the nature of this corporate form and links to cooperative principles. However, the mechanisms to perform that integration are varied. This paper analyzes the strengths and weaknesses of one of these formulas: merger. This analysis is based on the current situation of the agrifood cooperatives and legal incentives that these processes of concentration have, through the creation of so-called Priority Associative Entities and the provisions contained in the Act on measures to improve the food chain.

Key words: Cooperativa, merger, integration formula 


\section{La integración cooperativa como principio y los mecanismos de integración}

La cooperación entre cooperativas, consagrado como uno de los principios del movimiento cooperativo internacional, tiene sin duda una significado amplio, que excede el análisis de este trabajo. Ahora bien, es claro que dentro de ese acepción, general, pueden encontrarse sin duda, los mecanismos, contractuales o societarios, que permiten a las sociedades cooperativas crecer y lograr cotas más altas de competitividad. Y entre estos últimos, el procedimiento de fusión.

En efecto, lo que se pretende en estas líneas, es llamar la atención sobre las fortalezas y debilidades del proceso de fusión, que parece haber «renacido» en los últimos tiempos, sobre todo en el marco del cooperativismo agroalimentario. Y ese análisis de las fortalezas y debilidades sólo puede realizarse comparando tal procedimiento con otras fórmulas de integración cooperativa.

Entiéndase que cuando hablamos de integración cooperativa, lo hacemos en términos vagos, o más exactamente, de manera tan amplia que incluya todo un haz de procesos, internos y externos, contractuales y societarios, que permitan las sociedades cooperativas alcanzar de manera más óptima sus objetivos ${ }^{1}$.

En este sentido cabe recordar que estos procesos pueden conllevar el surgimiento de una «entidad» que personifique jurídicamente la integración ${ }^{2}$ o por el contrario que tal integración, más bien intercooperación, cristalice en una realidad económica de grupo, carente de personalidad jurídica ${ }^{3}$.

Todos los procesos de concentración ${ }^{4}$ tienen ventajas e inconvenientes, y presentan singularidades propias. Sin embargo parece que

1 Un estudio sobre las diferentes técnicas de integración cooperativa, puede verse en ALFONSO SÁNCHEZ, R, La integración cooperativa y sus técnicas de realización: la cooperativa de segundo grado, Tirant lo Blanc, 2000; sobre la dificultad también se ha manifestado. SÁNCHEZ PACHÓN, L., "La integración y la cooperación intercooperativas en el marco del derecho de defensa de la competencia», en La aplicación privada del derecho del competencia, AA.VV., Lex nova, 2011, págs. 937-953

2 Como puede ser la cooperativa de segundo grado.

3 El grupo cooperativo es así el instrumento por excelencia a través del que se intenta hacer realidad ese principio de intercooperación. Desde el punto de vista de «nacimiento» del grupo, es interesante señalar cómo el mismo trae causa de lo que bien podría conceptuarse como «contrato de grupo paritario». Sobre este tema puede verse el trabajo de EMBID IRUJO, J.M , «El contrato de constitución de grupo en el derecho español», RdS, n. ${ }^{\circ} 15,2000$,págs. 57 y ss.

4 Debe advertirse en este punto que la integración no se agota con instrumentos de derecho de sociedades, sino que tal integración, al menos económica, puede lograrse 
la realidad ha demostrado que en el marco del derecho cooperativo, la tendencia de integración se ha movido más en el marco de la constitución de grupos (personificados o no, en función de lo visto) que en el del recurso a la modificación estructural por referencia como es la fusión. La razón última podría estribar por un lado, en la existencia cierta de dificultades «técnicas» como se verá y por otro, por la pretendida «confrontación» de este mecanismo de integración con la propia naturaleza de las entidades mutualistas.

Sin embargo, lo que quiere señalarse en estas páginas es que esa tendencia parece querer cambiarse al menos, en el ámbito del cooperativismo agrario, en el que se han introducido medidas legislativas y sectoriales, encaminada ciertamente, de manera directa o indirecta, a fomentar la fusiones cooperativas. La duda que queremos plantear aquí es si ese «baile de cortejo» de cooperativas en búsqueda de alianzas, se ha abierto porque esa música agrada a las cooperativas hoy más que otra o si tiene que ver más bien con un mero escenario de promoción pública y de obtención de ventajas fiscales.

\section{La fusión de las cooperativas agrarias y las entidades asociativas prioritarias: ¿fomento ex lege u obligación?}

En efecto pues, el mejor escenario hoy para analizar el fenómeno de las fusiones de cooperativas es el de la integración en el marco del cooperativismo agroalimentario. En los últimos años se ha llevado a cabo un proceso de proliferación de normas e iniciativas que, desde lo poderes públicos, fomentan las alianzas con el objetivo de que las cooperativas de menor tamaño puedan alcanzar una dimensión adecuada para poder competir en el mercado. Así, el fenómeno de la fusión no es sólo una tendencia, sino que realmente se entiende con un plan de negocio.

Entre las normas que deben señalarse se encuentra en primer lugar la Ley 13/2013, de 2 de agosto, de fomento de la integración de cooperativas (en adelante LFIC) y de otras entidades asociativas de carácter agroalimentario, por la que se ha creado una nueva figura, la «Entidad Asociativa Prioritaria» (en adelante, EAP), cuyos requisitos para su reconocimiento han sido concretados a través del desarrollo reglamentario efectuado por el Real Decreto 550/2014, de 27 de junio (en adelante

de la mano de instrumentos contractuales, en los que los contratos llamados de distribución (franquicia, distribución exclusiva, ....) ocupan un lugar destacado. 
REAP). El objetivo de la norma no es otro que fomentar el crecimiento cuantitativo y dimensional de las pequeñas cooperativas agroalimentarias, dada la atomización del sector, para conseguir así unas cotas de competitividad respecto a otras figuras societarias, que puedan poco a poco equipararse a las cooperativas de ese sector en el ámbito europeo. Ese objetivo de promoción pretende conseguirse mediante el incentivo de la concesión, para quienes alcancen ese "título» de EAP, de una situación de preferencia en las ayudas de los Programas de Desarrollo Rural ${ }^{5}$.

Se da así, de la mano de esta norma, un empuje a la búsqueda de alianzas estratégicas para conseguir llegar a las dimensiones necesarias para obtener el reconocimiento de EAP. Ahora bien, la propia norma se mueve, de manera vaga, a la hora de determinar los mecanismos para lograr esas alianzas. En particular a lo largo de la norma se habla de fusión, de cooperativas de segundo grado y de grupos cooperativos. Se trata de alcanzar unos ratios cualificados en la norma en su desarrollo reglamentario ${ }^{6}$, no imponiendo un modo concreto para lograrlos. Logrados esos ratios, se deberá proceder al reconocimiento de la entidad «integrada» como EAP y es aquí donde queremos señalar algunas dudas que la regulación nos plantea. A la pregunta de quién puede ser reconocida como EAP, y por tanto, de qué mecanismos de integración serán los utilizados, la respuesta es amplia y, a nuestro juicio, carente de rigor jurídico. En efecto, y más allá de las dudas o problemas que se han señalado por la doctrina con relación a la equiparación de las a las SAT en el tratamiento dado a la integración de cooperativas ${ }^{7}$, entre el elenco de «entidades» que pueden obtener la calificación de EAP se nombran: las sociedades cooperativas agroalimentarias; las cooperativas de segundo grado; los grupos cooperativos; las organizaciones de productores con personalidad jurídica propia reconocidas de acuerdo con la normativa comunitaria en el ámbito de la Política Agraria Común y las entidades civiles o mercantiles —si son sociedades anónimas, sus acciones deberán ser nominativas-, siempre que más del 50 por ciento de su capital social pertenezca a sociedades cooperativas, a organizaciones de productores o a sociedades agrarias de transformación

5 Un interesante trabajo sobre esta figura, puede verse en CANO ORTEGA, C., «Hacia la calificación como Entidad Asociativa Prioritaria: las diferentes formas de integración de las cooperativas agroalimentarias», REVESCO, 2015, http://pendientedemigracion.ucm.es/info/revesco/txt/REVESCO\%20Cristina\%20CANO\%20ORTEGA.pdf

6 Ratios contenidos en el Anexo I Real Decreto 550/2014, de 27 de junio

7 Como señala CANO ORTENA, C., «Hacia...», pág. 6., resultado de su cuestionadas naturaleza que se debate entre la economía social y la del mercado. 
(art. 1.3 LFIC y art. 2.1 REAP). Esto es, esas entidades pueden ser calificadas de EAP y ser objeto del correspondiente registro. Ahora bien, nos parece difícil la conciliación de esta «personificación» con alguna de las realidades mencionadas, en particular, con la posibilidad de reconocer como EAP a u grupo cooperativo.

Aunque no es momento aquí para entrar al análisis detallado de lo que puede entenderse por grupo de sociedades ${ }^{8}$, sí es necesario recordar que el concepto de grupo, en general, y no sólo el de grupo cooperativo, es un concepto eminentemente económico, desde el momento en que la empresa, desde el punto desde el punto de vista económico, puede definirse como mera actividad. Sin embargo, no puede decirse que el grupo, por constituir empresa en sentido económico, pueda definirse como titular de esa actividad. Dentro de la noción jurídica de empresa aparecen diversos aspectos o perfiles claramente diferenciados. Entre ellos puede afirmarse que, desde el prisma del Derecho, debe atenderse a la dimensión «subjetiva» del fenómeno empresarial9.

Así, la empresa —en sentido jurídico-, requiere un sujeto al cual sea imputable su actividad. El empresario en sentido económico es, sin duda, el grupo (así, es más correcto hablar de «empresa de grupo», que de "grupo de empresas»), y ello porque el grupo no está constituido por la suma aritmética de muchas empresas, sino que constituye en sí mismo una «superempresa», con su propio interés, el interés del grupo.

Ahora bien, no se puede identificar al grupo con el titular de la actividad empresarial que realiza. Es decir, no se puede dotar al grupo de personalidad jurídica, y ello, no sólo por respeto de su propio carácter híbrido ${ }^{10}$, sino porque los postulados en que se asienta el Derecho de sociedades, y que hacen surgir a la sociedad, como persona jurídica, diferente a los socios que la crean, no pueden ser aquí de aplicación.

8 Un recorrido exhaustivo de la doctrinad de los grupos puede verse en nuestro trabajo El proceso de constitución de la Sociedad Europea Holding, Aranzadi, 2008, . y en la bibliografía allí recogida.

9 FERNÁNDEZ DE LA GÁNDARA/GALLEGO, E. Fundamentos de Derecho mercantil I, Valencia, 1999, p. 122. Sobre el concepto de empresa, vid. entre otros GONDRA ROMERO, J.M., «La estructura jurídica de la empresa (El fenómeno de la empresa desde la perspectiva de la Teoría General del Derecho)», RDM, n. ${ }^{\circ} 228,1998$, pp. 493 y ss.

10 En este sentido ANTUNES,, E. Os grupos de sociedades, 2. ${ }^{a}$ Edición, Coimbra, 2002 p. 156. que habla de la personificación del grupo como su propio «homicidio jurídico». 
Debe recordarse también, que cuando hablamos de grupo cooperativo hablamos de lo que la doctrina define como grupo "paritario» ${ }^{11}$. En el caso de los grupos cooperativos, regulados en el art. 78 de la LCoop., la peculiaridad del nacimiento del grupo radica en el mismo deviene de la voluntad "contractual» de las sociedades cooperativas que quieren integrarse. Se da así una coincidencia temporal entre la formación del grupo, en sentido económico y su efectiva constitución jurídica mediante el oportuno contrato. Dado que ninguna sociedad está dominada por otra en dicho grupo -ya que en consonancia por la propia naturaleza "democrática» de la cooperativa, no cabe ese control—, el ejercicio de la dirección unitarias — de unidad de decisión habla la LCoop- se articula en el propio documento contractual, que se verá reflejado en los Estatutos de las sociedades del grupo, debiendo procederse a la anotación del acuerdo — de competencia asamblearia - en la hoja registral de cada sociedad integrada.

Siendo esto así, la pregunta que nos hacemos es si ciertamente una de las vías para obtener el reconocimiento como EAP será la constitución de grupos cooperativos. Se nos hace complicado, y no sólo por lo expuesto y que como se ha visto entronca con la necesidad de cierta «personificación» de esa integración, sino porque difícilmente un grupo cooperativo que pretenda la catalogación como EAP, podrá presentar, tal y como establece el artículo del REAP, a través de su representante legal (que no lo tiene) los Estatutos o disposiciones reguladoras de la entidad (recordemos, grupo cooperativo que quiere obtener el reconocimiento de EAP, que por tanto, carece de Estatutos).

Más fácil será que la integración promovida por la LFIC se lleve a cabo a través de la constitución de cooperativas de segundo grado o del procedimiento de fusión. En efecto, la única EAP reconocida hasta la fecha de elaboración de este trabajo, la cooperativa OVIESPAIN ${ }^{12}$, es una sociedad cooperativa de segundo grado ${ }^{13}$. Mientras que existe intención

11 Nos remitimos a los trabajos, entre otros de, SACRISTÁN REPRESA, M., «El grupo de estructura paritaria: caracterización y problemas», RDM, n. ${ }^{\circ} 165-166,1982$, pp., 375 y ss; FERNÁNDEZ DEL POZO, L., "Grupos horizontales transfronterizos. Un primer estudio sobre las sociedades gemelas», RDM, n. ${ }^{\circ} 235$, enero-marzo 2000, pp. 147 y ss; EMBID IRUJO, J.M, «Problemas actuales de la integración cooperativa, $R D M, \mathrm{n} .{ }^{\circ} 227,1998, \mathrm{pp} .7$ y ss.

12 http://www.magrama.gob.es/gl/prensa/150406gtejerinareconocimientooviespai n_tcm10-371089_noticia.pdf

13 Ovispain está formada por EA Group (integrada por las cooperativas de segundo grado Oviso de Extremadura y Cordesur, de Andalucía Occidental); la cooperativa Cosegur, que reúne a productores de la Raza Segureña, de Andalucía Oriental, Murcia y Castilla La Mancha; y Oviaragón, cooperativa ganadera de ovino líder en Aragón y principal productora de Ternasco de Aragón I.G.P. Un total de 4.300 ganaderos de ovino que 
de solicitar reconocimiento como EAP multisectorial de entidades resultado de fusiones, como la llevada a cabo por DCoop, y Baco ${ }^{14}$.

La pregunta es cuál de estos mecanismos (concentración vía cooperativa de segundo grado o fusión) será el preferido en estos movimientos de integración ${ }^{15}$. Sólo un análisis de los problemas que uno y otro plantea, y que se hará en los epígrafes que siguen, puede arrojarnos algo de luz.

Para concluir con este análisis de por qué en los últimos años se ha producido movimiento entre las cooperativas en búsqueda de integraciones y antes de analizar las ventajas e inconvenientes de las diferentes formas de integración, quiere señalarse que no sólo la LFIC coadyuva la intención de procurar la concentración de las sociedades cooperativas. Es en el ámbito de la Ley 12/2013, de 2 de agosto, de medidas para mejorar el funcionamiento de la cadena alimentaria (LMMFCA), establece, como una de las prioridades de la norma, fomentar una mayor integración de los operadores que intervienen en la cadena alimentaria, con objeto de facilitar una mayor eficiencia y rentabilidad en los distintos sectores que la integran. Estableciendo la conveniencia de que los operadores de la cadena se sometan a un Código de Buenas Prácticas. Sobre lo que se volverá.

\section{Principales «problemas» de la fusión de cooperativas}

\subsection{La relación de canje y a patrimonialización de la posición del socio}

Analizadas en líneas anteriores, las claves, normativas y de política jurídica, que pueden explicar el por qué de una dinamización de las operaciones de integración en el ámbito cooperativo, especialmente, en el de las cooperativas agroalimentarias, se quieren recodar y siste-

gestionan en torno a 1,4 millones de ovejas, lo que supone cerca del $15 \%$ del total de la cabaña de ovino de carne española.

14 http://www.magrama.gob.es/gl/prensa/noticias/fernando-burgaz-subrayala-necesidad-de-promover-un-modelo-cooperativo-m\%C3\% A1s-potente-endimensi\%C3\%B3n-ambici\%C3\%B3n-innovadora-y-proyecci\%C3\%B3n-internacional-/ tcm10-345143-16

15 Un interesante trabajo de análisis comparativo de estas formas de integración, puede verse en MELIÁ MARTÍ/FERNANDO LUCAS/JORGE FARIA, «Cooperativas de segundo grado versus fusión. Ventajas e inconvenientes» en Integración empresarial cooperativa : posibilidades, ventajas e inconvenientes: [ponencias del II Coloquio Ibérico de Cooperativismo y Economía Social] / coord. por Rui Namorado, Rafael Chaves Ávila, Isabel Gemma Fajardo García, 2003, págs. 105-142 
matizar ahora, cuáles han sido tradicionalmente, los problemas jurídicos con que estas operaciones se han encontrado, particularmente, cuando se han incordiando en el ámbito de las fusiones.

Debe recordarse, de manera sucinta, que la fusión de cooperativas puede definirse - utilizando el concepto aplicable a la fusión de las sociedades de capital (art. 22 Ley de modificaciones estructurales- LME - dada la ausencia de definición de fusión del la LCoop- como proceso en virtud del cual, dos o más sociedades que se extinguen, transmiten su patrimonio en bloque a una nueva sociedad que se constituye a estos efectos (fusión por creación de nueva sociedad), o a una de ellas (fusión por absorción), pasando generalmente sus socios a la sociedad que se constituye o a la sociedad absorbente en función del negocio que todas ellas asumen. Los motivos señalados por la doctrina del raro uso de la fusión por parte de las cooperativas se deben principalmente a doble hecho de que el rendimiento económico de las cooperativas no está en proporción directa con la concentración e inversión en capital, sino con el incremento de la actividad cooperativizada de sus socios y la propia naturaleza de las cooperativas como sociedades de capital variable y de libre adhesión o de puertas abiertas ${ }^{16}$.

Ahora bien, más allá de esos problemas que parte de la doctrina enlaza a la naturaleza de la sociedad cooperativa ${ }^{17}$, y que creemos deben superarse dado el devenir de esta figura societaria y su necesidad de ser "competitiva» con otras formas jurídicas, son problemas técnicos, sustantivos, los que dificultan al recurso a este modo de integración. Problemas como la determinación de la relación de canje o el reconocimiento del derecho de separación.

Dado que señala la LCoop. en el apartado 4 del art. 64, la obligatoriedad de establecer una «relación de canje» en las fusiones de cooperativas $^{18}$, al señalar como contenido del proyecto de fusión el sistema

16 MACÍAS RUANO, A.J, "Modificaciones estructurales en las cooperativas y sociedades agrarias de transformación», en PULGAR EZQUERRANARGAS VASSEROT (Coord). Cooperativas agrarias y sociedades agrarias de transformación., Madrid, Dykinson, págs. 679 y ss.

17 De opinión contraria sin embargo, se manifestaba SANZ JARQUE, J.J., Cooperación. Teoría general y régimen de las sociedades cooperativas. El nuevo derecho cooperativo, Comares, Granada, 1994, pág. 595, al afirmar que la fusión «es consustancial a la naturaleza de las mismas y del movimiento cooperativo, y es una consecuencia del principio de integración cooperativa».

18 Pues entendemos que si bien con peculiaridades, sí hay una relación de canje también en el ámbito de las fusiones íntercooperativas. En opinión de MACÍAS RUANO, A.J., op cit. pág. 690, sin embargo, «no existe en el ámbito cooperativo, ni la relación ni el procedimiento de canje.... y ello porque la aportación no es medida de los derechos del socio cooperativista». 
para fijar la cuantía que se reconoce a cada socios de las cooperativas que se extingan como aportación al capital de la cooperativa nueva o absorbente, debe recordarse siquiera brevemente qué debe entenderse por tal relación de canje.

Pues bien, la doctrina sobre fusiones, viene a definirla, con unos u otros términos como elemento técnico mediante el cual se puede atribuir a los socios de las sociedades intervinientes en determinados procesos de reestructuración societaria participación de una nueva sociedad $^{19}$. Este tipo de canje debe permitir conocer la exacta medida de esa participación que corresponde a cada socio en la sociedad resultante. Y a tales efectos, como se ha dicho en sede de fusiones de sociedades de capital, será preciso realizar dos cálculos diferentes en su objeto: el primer cálculo traduciría la relación que existe entre todas las sociedades participantes en el proceso de fusión ${ }^{20}$, el segundo concreta la particular relación existente entre lo «transmitido» por parte cada una de las sociedades y el capital total de la sociedad resultante. El tipo de canje por tanto resultante expresará una doble relación de proporcionalidad: una «externa» en atención a la comparación de las sociedades y otra «interna», relativa a los criterios de reparto del capital de sociedad resultante que corresponde asignar a cada socio ${ }^{21}$.

No podemos entrar aquí a analizar la polémica que, en sede de fusiones de sociedades de capital, se ha dado a la hora de entender si tal operación de fijación está fuera de la autonomía contractual de las sociedades y constituye una declaración de la mayoría de contenido predeterminado por la Ley o si por el contrario, la misma incluye elementos de negociación o incluso, si el tipo de canje es el «precio de la operación» y por ello de libre fijación por las partes. Ahora bien, no cabe duda que debe tenderse, también en el ámbito de las fusión de cooperativas a una relación de canje que concilie de alguna forma los intereses en juego. En primer lugar el propio interés de los socios ${ }^{22}$ que en atención a los principios que rigen el contrato de sociedad, tenderá

19 Así lo define, con referencia, a las sociedades anónimas, SEQUEIRA MARTíN, A., en SÁNCHEZ CALERO, F., (Dir), Comentarios a la Ley de Sociedades Anónimas, Tomo VII: Transformación, Fusión y escisión, arts. 223 a 259, Madrid, 1993, pp, 79 y sS, p. 135.

20 Se entiende esta globalidad referida el porcentaje fijado en el proyecto para cada una de las sociedades participantes.

21 Con relación a la fusión de sociedades de capital, así lo señala, PÉREZ TROYA, A., La determinación del tipo de canje en la fusión de sociedades, Madrid, 1998, pp. 71 a 73 .

22 Que en última instancia aprobarán la relación de canje «ofertada» por el órgano de administración. 
en la determinación de la relación de canje a la maximización del valor de su participación. En segundo, el propio de los acreedores, en la medida en que en esa determinación de la relación de canje deberá velarse también por el cumplimiento de determinados principios de capital y que hacen referencia, especialmente al principio de realidad. ${ }^{2324}$

El problema radica principalmente en establecer si debe reconocerse a los socios de las sociedades cooperativas participantes en la fusión, capital en el sociedad absorbente a cuenta del patrimonio resultante, teniendo en cuenta que tiene su origen en una revalorización de los activos o sólo a cuenta del patrimonio repartible. Siguiendo a parte de la doctrina 25 , debería defenderse que la cooperativa resultante de la fusión emita capital únicamente por aquella parte del patrimonio de las cooperativas que se fusionan que sea repartible al socio (capital social y fondos de reserva voluntarios repartibles, principalmente, de cada cooperativa). Y en el caso del patrimonio originado en la actualización del balance para la fusión, que se le asigne un carácter repartible o no ${ }^{26}$, según el carácter d ella partida patrimonial en la que se integraría el resultado que generaran si no habiéndose producido la supón se enajenaran, y afloraran realmente las plusvalías.

En definitiva pues, la especialidad en cuanto a la determinación de la relación de canje, deriva de la dificultad de «patrimonializar» la posición de socio cooperativo. Sin embargo, lo que sí es interesante es volver a insistir en la posibilidad de cierta modulación en la determinación de esa relación de canje de la operación de fusión ${ }^{27}$, así como en la ne-

23 En este sentido, como señala MELIÁ MARTí, E., «El derecho de separación del socio cooperativista en la fusión de cooperativas. Efectos de la reforma de la norma contable en estos procesos», en Revista Española de Estudios Agrosociales y Pesqueros, n. ${ }^{\circ} 217,2008$, págs, 105 y ss., pág. 105, si bien la norma cooperativa no concreta si el capital social que se va a reconocer en la cooperativa resultante a los socios de las diferentes cooperativas implicadas debe ser cualificado a partid d ellos patrimonios contables de las cooperativas, o si por el contrario debe ser calculado en términos reales, debe optarse por entender que es siempre deseable establecer en términos reales tanto el capital a reconocer como las compensaciones en metálico que haya que efectuar para compensar desequilibrios patrimoniales.

24 Especial interés tiene la vertiente económico-contable de las fusiones, también apea entender la relación de canje, Vid por todo, FERNÁNDEZ DEL POZO, L., «Derecho contable de fusiones y escisiones», Marcial Pons, 2008.

25 MELÍA MARTí, E., op, cit., pág. 121

26 Un análisis acertado del problema puede verse también en ALONSO, E., «La fusión, la escisión, la transformación y la extinción de las cooperativas», en Boletín de la Asociación Internacional de Derecho Cooperativo , n. ${ }^{\circ} 34,2000$, pág. 75-114., esp. págs. 79 y 80 .

27 En esa idea ya expresada de necesidad de conciliar, en la determinación del precio de la operación, los intereses en juego, a la vista de la obtención de sinergias. 
cesidad de enlazar la «razonabilidad» o no de esa modulación, con el hipotético derecho de separación reconocido a ese socio.

\subsection{El derecho de separación: su fundamento y la analogía con su utilización en las sociedades de capital}

A diferencia de lo que ocurre con la fusión de sociedades de capital (con la excepción de las fusiones transfronterizas ex art. 62 LME), donde los socios de las sociedades participantes en la operación no tienen reconocido del derecho de separación, el art. 65 de la LCoop. establece que sí se reconoce el mismo a los socios de las sociedades cooperativas que no hubieran votado a favor.

No queremos reproducir ahora el debate doctrinal sobre quiénes tienen tal derecho ${ }^{28}$, cómo puede ejercerse ${ }^{29}$ y cómo debe liquidarse el mismo ${ }^{30}$, remitiéndonos a la doctrina que ha tratado profusamente el tema, pero sí intentar analizar la justificación y racionalidad de tal derecho en el marco del objetivo de nuestro estudio, que no es otro, que el fenómeno de promoción de operaciones de integración cooperativa en el marco, principalmente de cooperativismo agroalimentario.

Se ha dicho por parte de la doctrina que el derecho de separación en el ámbito cooperativo no es un tipo de baja voluntaria, derivada del principio de puertas abiertas y capital variable de las sociedades cooperativas, sino una clase distinta ${ }^{31}$. No obedecería así a la simple voluntad del socio, sino que deriva y nace, en este caso, de un específico supuesto regulado en la norma. En este sentido, la baja está justificada ex lege. Y nos preguntamos, ¿existe ciertamente tal justificación? ¿razonable el reconocimiento del derecho de separación en la fusión de sociedades cooperativas? ¿qué protege ese derecho?

28 Esto es, cómo debe entenderse por «socio disconforme» o si también debe reconocerse a los socios inactivos, excedente, asociados o colaboradores. vid. por todos MELIÁ MARTí, E., op. cit. pág. 109-111.

${ }_{29}$ Cfr. apartado 1 art. 65 LCoop. mediante escrito dirigido al Presidente del Consejo Rector, en el plazo de cuarenta días desde la publicación del anuncio del acuerdo, según lo previsto en esta Ley

30 Cfr. apartado 2 art. 65 LCoop. «La cooperativa resultante de la fusión asumirá la obligación de la liquidación de las aportaciones al socio disconforme, en el plazo regulado en esta Ley para el caso de baja justificada y según lo establecieran los Estatutos de la cooperativa de que era socio»

31 En sentido contrario MACÍAS RUANO, A.J., op cit. pág. 695, para quien el derecho de separación en las sociedades cooperativas no pueden entenderse sino como el derecho de baja voluntaria. 
El objetivo de este trabajo no es otro que intentar poner sobre la mesa las implicaciones y dudas que plantea el movimiento de integración cooperativa «promovido» si no, «forzado» por algunas normas que buscan la dinamización, principalmente del sector agroalimentario, y la consecuención de cotas dignas de competitividad en el mercado de las pequeñas cooperativas, en un mundo totalmente atomizado. Ahora bien, ese loable interés, y por tanto, la proliferación de procesos de constitución de grupos cooperativos, cooperativas de segundo grado o, en lo que ahora nos ocupa fusiones, no puede obviar la razón última de su repunte: crear sinergías y conseguir competitividad.

Optar por utilizar el mecanismo de la fusión como vía de integración para obtener esas sinergías, es contar con la posibilidad de que la operación quede frustrada si los socios de las sociedades implicadas ejercitan su derecho de separación, a menos que intentemos encuadrar de algún modo la justificación de tal ejercicio, en el marco del interés general en la integración. ${ }^{32}$

Sería algo así como proponer, que la justificación del derecho de separación podría verse en el miedo a la integración. Yendo más allá, que sólo debería reconocerse ese derecho de separación si esa integración abre la posibilidad de perjudicar la posición del socio, lo que sólo podría acontecer, en términos de grupos, si ese socio es externo.

El derecho de separación tendría así una connotación diferente en sede de fusiones, donde quizá debería relacionarse con la razonabilidad en la determinación de la relación de canje (convirtiendo un mecanismo ex post, como sería la impugnación de la fusión por una inadecuada determinación de la relación de canje, en un mecanismo de protección del socio ex ante) y en el ámbito de las cooperativas de segundo grado y los grupos cooperativos, donde ese derecho de separación vendría reconocido por la integración en el grupo, de forma similar a lo propuesto en el Proyecto de Código Mercantil,

32 En este sentido la reflexión, que compartimos, de DE CARLOS/ RIPLEY/ VENTOSO/ BAGARÍA, «Procesos de reorganización empresarial: algunas propuestas de leve ferenda», http://www.uria.com/documentos/publicaciones/3208/documento/art24.pdf?id=3353 quienes, en el marco del análisis del reconocimiento o no del derecho de separación cuando en el marco de una fusión de sociedades de capital se producen modificaciones estatutarias que sí reconocen ese derecho, abogan por una solución coherente con el favorecimiento de las operaciones de concentración y, con ello, con la consolidación de estructuras sólidas en situaciones de crisis, pase por dar prevalencia al interés social sobre al interés particular del socio en los casos de fusión, para evitar la frustración de operaciones de consolidación articuladas a través de la operación de fusión, evitando la consecución de objetivos de concentración de capital de gran relevancia. 
en cuyos artículos 291-15 y 291 16, se regulan, respectivamente, la figura del socio externo en el grupo y el reconocimiento del derecho de separación de ese socio externo por la integración en un grupo. Ahora bien, en este último caso se abre un problema añadido y es que es difícil encajar la figura del socios externo en el ámbito cooperativo, toda vez que la noción de control y dominación no se dan en el fenómeno de la integración entre cooperativas. Según lo señalado líneas antes. ${ }^{33}$

\subsection{Las cuestiones del derecho de la competencia}

Como último estadio en este análisis de los problemas jurídico mercantiles que deben tomarse en consideración a la hora de fomentar la integración cooperativa, quiere hacerse una mención, bien será somera, a la afectación de este fomento en el ámbito de la defensa de la competencia.

La defensa a ultranza de una «competencia perfecta» puede implicar una serie de costes sociales que muy posiblemente no siempre resultarán compensados por los beneficios de ella, por lo que su aspiración tendrá que limitarse a la consecución de una competencia suficiente $^{34}$. Así lo ha debido pensar el legislador al promulgar las dos normas mencionadas al comienzo de este trabajo y que promueven, sobre todo en el sector del cooperativismo agroalimentario, la operaciones de integración.

Sin embargo, la integración no puede hacerse a toda costa, o al menos, o a costa de que esa competencia, quede cercenada, más allá de lo razonable.

En este sentido sólo quieren señalarse aquí los dos informes que la Comisión Nacional de la Competencia y los Mercados ha hecho sobre las dos normas mencionadas la LFIC y la LMMFC.

33 Y ello sin perjuicio de que, ya, en el funcionamiento grupal, puedan producirse algún perjuicio de la sociedad integrada en un grupo cooperativo, sin la compensación adecuada, y dar lugar al reconocimiento de nuevo del derecho de separación (así lo establece el art. 291-17 del proyecto de Código Mercantil). Toda vez que, la búsqueda del interés de grupo es consustancial no sólo a los grupos por subordinación, sino también a los grupos por coordinación, donde, según lo señalado líneas antes, se incordian el grupo cooperativo, y en esa búsqueda del interés del grupo, se pueda producir un perjuicio individual a una de las sociedades agrupadas. Pero esto es un supuesto diverso del analizar, que lleva a planteamientos complejos que tienen que ver con la doctrina de las ventajas compensatorias.

34 SÁNCHEZ PACHÓN,L. op cit., pág. 947. 
Con relación a la primera norma, recuerda la $\mathrm{CNMC}^{35}$ que la misma contiene medidas que privilegian a unas estructuras productivas agroalimentarias (las entidades asociativas preferentes) frente a otras: aquellas entidades que, pudiendo optar por esta integración, decidan no hacerlo, y también otras formas jurídicas que escapan al ámbito de aplicación de la Ley (por ejemplo, sociedades mercantiles cuyo capital mayoritario no está en manos de cooperativas). Existiendo, por tanto, una voluntad de condicionar con estas medidas la morfología de la futura estructura de la oferta, debe extremarse la exigencia de justificar adecuadamente la necesidad y la proporcionalidad de las medidas, para asegurar que la selectividad de las mismas no condiciona indebidamente la competencia en el mercado ${ }^{36}$.

No se trata tanto, señala la CNMC, de buscar la justificación de las medidas incluidas en la hoy LFIC remitiéndose a las discusiones mantenidas años atrás y relativas a los beneficios de que disfruta la figura de la cooperativa, y que «legalizaban» el tratamiento fiscal diferenciado. Y ello, porque en efecto, el ámbito subjetivo de entidades que pueden optar a la condición de entidad asociativa prioritaria, de acuerdo con la Ley, tampoco se limita a las sociedades cooperativas en sentido estricto. La justificación de la norma entronca con su verdadera utilidad para lograr el objetivo planteado, que no es otro que incrementar el tamaño y escala de estos operadores, fundamentalmente dirigidas a la constitución y puesta en marcha de las EAP, y no a establecer ventajas para ellas de ámbito temporal indefinido para su funcionamiento operativo. En particular cuando se trata de medidas que privilegian el acceso a financiación pública o al disfrute de otros activos públicos.

Por lo que respecta a las medidas de fomento de la integración cooperativa contenidas en la $\operatorname{LMMFC}^{37}$ y de sus normas de desarroIlo, una de las cuestiones que sobre las que la CNMC plantea alguna reserva es la relativa a la prioridad para recibir ayudas y subvenciones del Ministerio de Agricultuda, a los solicitantes adheridos al llamado Código de Buenas Prácticas Mercantiles en la Contratación Alimentaria (CBPMCA). Tal código, que contiene las prácticas mercantiles que fomenten relaciones justas, equilibradas y leales entre los operadores de la cadena alimentaria, y al que se pueden adherir de manera vo-

35 Informe IPN 82/12. Anteproyecto de Ley de Fomento de la Integración Cooperativa y Asociativa.

${ }_{36} \mathrm{Cfr}$. páginas 5 y 6 del informe

37 http://www.cnmc.es/Portals/0/Ficheros/Promocion/Informes_sobre_normativa/2014/140715_IPN_DP_0009_14_\%20PRD\%20cadena\%20alimentaria.pdf 
luntaria los operadores, se presenta como un instrumento para corregir fallos del mercado, corrección a la que coadyuva la concesión de esas ayudas o subvenciones. Ahora bien, como señala la CNMC, el desequilibrio o la asimetría entre las partes contratantes no es una anomalía o un fallo del mercado sino una característica inherente a él. A este desequilibrio los operadores participantes en el mercado van dando respuesta desde un punto de vista dinámico, adaptando su organización productiva y mejorando su eficiencia. Ello contribuye a mejorar la competitividad y productividad de estos sectores, a fomentar la innovación, y a fortalecer en definitiva a los propios sectores en los que se generan estas tensiones. En este sentido es claro que cualquier intervención pública que limitase la libre voluntad de los operadores de suscribir un el código de buenas prácticas, no superaría un juicio de ponderación de la necesidad y proporcionalidad ni de mínima restricción competitiva de la misma, por cuanto no producirá incrementos de eficiencia del mercado ni aumentaría las tensiones competitivas entre los propios operadores, en perjuicio del interés general.

Cuestión diferente son los interesantes debates que la irrupción de este CBPMCA plantea en el ámbito de la integración y de la responsabilidad o imputación de conductas desleales en el seno de los grupos. Interesantes debates, que exceden sin duda el objetivo humilde de este trabajo.

\section{Conclusiones}

Que debe fomentarse la integración cooperativa, no es discutible. Que en el ámbito de las cooperativas agroalimentarias tal integración se presenta como la única vía de poder contar en nuestro ordenamiento con entidades competitivas en el mercado europeo, tampoco. Ahora bien, los protagonistas del baile de cortejo no deben olvidar, en primer lugar, que los mecanismos para lograr acercarse a las cuotas de crecimiento necesario para ser reconocidas como EAP, son variados, como variados son los problemas que presentan; y en segundo lugar, que la integración debe entenderse como un relación duradera, por lo que deben contarse con los «inconvenientes» que esa relación pueda traer.

Porque no es lo mismo fusionarse que constituir una sociedad de segundo grado, o incorporarse a un grupo cooperativo, por más que, a los efectos de la norma, sirvan todas estas figuras (o pseudofiguras, según lo visto en la crítica a la falta de rigor a la hora de abrir la puerta a 
la posibilidad de creación de las EAP). Y no es lo mismo, porque, mientras en la fusión son los problemas de la relación de canje o el derecho de separación, algunos de los aspectos, que tradicionalmente, y también hoy, pueden hacer menos atractivo el recurso a la modificación estructural por excelencia, en el ámbito de las cooperativas de segundo grado y de los grupos cooperativos, son las cuestiones grupales, entre las que se encuentran, cuestiones de responsabilidad y reparto de riesgos, las que no pueden olvidarse.

Y ello al margen del recorrido que estas normas y su aplicación tenga en el ámbito del derecho de la competencia.

Crecer por qué, para qué, y a qué coste. Esa es la cuestión.

Sólo el tiempo dirá si los cortejos llegan a fructificar en muchos enlaces, y si, pasado el tiempo de las vacas flacas, en el que la promoción pública alienta con el incentivo fiscal a los operadores, las integraciones del sector agroalimentario se mantienen fuertes por haber logrado encontrar el equilibrio justo entre competitividad y la lealtad a los principios cooperativos.

\section{Bibliografía}

Alfonso SÁnchez, R, La integración cooperativa y sus técnicas de realización: la cooperativa de segundo grado, Tirant lo Blanc, 2000.

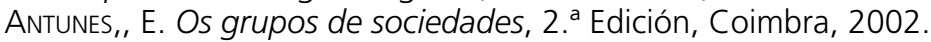

Cano Ortega, C., «Hacia la calificación como Entidad Asociativa Prioritaria: las diferentes formas de integración de las cooperativas agroalimentarias», REVESCO, 2015.

EMBID IRUJO, J.M, "Problemas actuales de la integración cooperativa, RDM, n. ${ }^{\circ} 227,1998$, pp. 7 y ss.

- «El contrato de constitución de grupo en el derecho español», $R d S, \mathrm{n} .{ }^{\circ} 15$, 2000, pp. 57 y ss.

Fernández De La Gándara/Gallego, E. Fundamentos de Derecho mercantil I, Valencia, 1999

Fernández Del Pozo, L., «Grupos horizontales transfronterizos. Un primer estudio sobre las sociedades gemelas», RDM, n. ${ }^{\circ} 235$, enero-marzo 2000, pp. 147 y ss.

— «Derecho contable de fusiones y escisiones», Marcial Pons, 2008.

GondRa Romero, J.M., "La estructura jurídica de la empresa (El fenómeno de la empresa desde la perspectiva de la Teoría General del Derecho)», RDM, n. ${ }^{\circ} 228,1998$, pp. 493 y ss.

Navarro LÉrida, M. ${ }^{a}$ S. El proceso de constitución de la Sociedad Europea Holding, Aranzadi, 2008.

MaCíAS RUANO, A.J, "Modificaciones estructurales en las cooperativas y sociedades agrarias de transformación», en Pulgar EzQueRraNARgas VASSEROT (Coord). 
Cooperativas agrarias y sociedades agrarias de transformación., Madrid, Dykinson, pp. 679 y ss.

Melí Marti/Fernando LuCAS/JORGe Faria, "Cooperativas de segundo grado versus fusión. Ventajas e inconvenientes» en Integración empresarial cooperativa : posibilidades, ventajas e inconvenientes : [ponencias del II Coloquio Ibérico de Cooperativismo y Economía Social] / coord. por Rui Namorado, Rafael Chaves Ávila, Isabel Gemma Fajardo García, 2003, pp.. 105-142.

MELIÁA MARTÍ, E., «El derecho de separación del socio cooperativista en la fusión de cooperativas. Efectos de la reforma de la norma contable en estos procesos», en Revista Española de Estudios Agrosociales y Pesqueros, n. ${ }^{\circ} 217$, 2008, pp, 105 y ss.

PÉRez Troya, A., La determinación del tipo de canje en la fusión de sociedades, Madrid, 1998

SACRISTÁN REPRESA, M., «El grupo de estructura paritaria: caracterización y problemas», RDM, n. ${ }^{\circ} 165-166,1982$, pp., 375 y ss.

SÁNCHEZ PACHÓN, L., "La integración y la cooperación intercooperativas en el marco del derecho de defensa de la competencia», en La aplicación privada del derecho del competencia, AA.VV., Lex nova, 2011, pp.. 937-953.

SANZ JARQUE, J.J., Cooperación.Teoría general y régimen de las sociedades cooperativas. El nuevo derecho cooperativo, Comares, Granada, 1994.

Sequeira Martín, A., en Sánchez Calero, F., (Dir), Comentarios a la ley de Sociedades Anónimas, Tomo VII: Transformación, Fusión y escisión, arts. 223 a 259, Madrid, 